\title{
Paludisme grave chez un nouveau-né prématuré à l'unité de néonatologie de l'Hôpital du Mali
}

\section{Severe malaria in a premature newborn at the Neonatology Unit of the Mali Hospital}

\author{
Kané $\mathbf{B}^{1}$, Diallo $\mathrm{KW}^{1}$, Koné $\mathrm{AK}^{5}$, Simaga $\mathrm{T}^{1}$, Koné $\mathrm{O}^{3}$, Traoré $\mathrm{A}^{4}$, Dicko $\mathrm{FT}^{2}$. \\ 1- Service de pédiatrie de l'Hôpital du Mali \\ 2- Service de pédiatrie du CHU Gabriel Touré \\ 3. Institut National en Santé Publique \\ 4- Service de Gynéco-obstétrique de l'Hôpital du Mali \\ 5. Centre de recherche et de formation sur le paludisme / FMOS/USTTB, Mali.
}

Auteur correspondant : Dr Bourama Kané, Service de pédiatrie de l’Hôpital du Mali. E-mail : bkanebassidiki.bk@gmail.com

\section{Résumé}

Introduction : le paludisme est un problème de santé publique au Mali. Cependant il est si rare chez le nouveauné que sa recherche n'est pas systématique chez ce dernier même fébrile.

Observation : il s'agissait un nouveau-né prématuré de 21 jours admis pour fièvre à la néonatologie de l'Hôpital du Mali. Le diagnostic a été posé au test rapide (TDR) du paludisme. Un traitement comprenant l'artésunate, la transfusion de concentré globulaire et la perfusion du sérum glucosé $10 \%$ a été institué. L'évolution a été marquée par l'apyrexie, la reprise de l'alimentation et du poids, la diminution de la parasitémie et l'augmentation du taux de l'hémoglobine.

Conclusion : le TDR du paludisme doit être systématique chez les nouveau-nés fébriles en zone d'endémie palustre pour ne pas passer à côté d'un paludisme néonatal.

Mots clés : Paludisme, nouveau-né, hôpital du Mali.

\section{Summary}

Introduction: malaria is a public health problem in Mali. However, it is so rare in the newborn that its search is not systematic in the latter even feverish.

Observation: this was a 21-day premature newborn admitted for fever at the neonatology department of the Mali Hospital. The diagnosis was made with the rapid test (RDT) for malaria. A treatment including Artesunate, transfusion of globular concentrate and infusion of $10 \%$ glucose serum has been initiated. The evolution was marked by apyrexia, resumption of diet and weight, decrease in parasitaemia and increase in hemoglobin level.

Conclusion: the RDT of malaria must be systematic in febrile newborns in the malaria endemic zone so as not to miss neonatal malaria.

Keywords: Malaria, newborn, Mai hospital.

\section{Introduction}

Le paludisme est une maladie due à un parasite du genre Plasmodium transmis par des piqûres des moustiques femelles du genre Anophèle[1].Le paludisme est une endémie parasitaire majeure et constitue encore de nos jours un problème de santé publique dans le monde. Chaque année l'Organisation Mondiale de la Santé (OMS) estime à plus de 200 millions le nombre de cas de paludisme dans le monde avec plus de 400000 décès. Environ $90 \%$ de ces décès imputables à cette maladie se produisent en Afrique subsaharienne tandis que le reste de la mortalité se répartit entre l'Asie du Sud-Est, l'Amérique du Sud, le Pacifique occidental et la Méditerranée orientale[2].Au Mali, le paludisme est la première cause de consultation, d'hospitalisation et de mortalité dans les formations sanitaires. Selon l'Annuaire Statistique du Système Local d'Information Sanitaire (SLIS) en 2014 les formations sanitaires ont enregistré plus de 2.500000 cas suspects de paludisme soit $40 \%$ de motifs de consultation dont environ 1700000 cas simples et 800000 cas graves avec 2309 décès[3]. Cependant le paludisme du nouveau-né est une affection rare même dans les zones d'endémie palustre. En 2013 une étude menée au Centre Hospitalier Régional de Ziguinchor sur le paludisme congénital a révélé une fréquence de 1.05\%[4]. En 2013 une étude menée au Centre Hospitalier Universitaire Souro SANOU de BoboDioulasso a montré une prévalence de $0,42 \%$ de paludisme congénital[5].Au Mali, une étude réalisée sur le paludisme chez le nouveau-né en 2008 au service de néonatologie du CHU Gabriel Touré n'a trouvé aucun cas de paludisme congénital[6].Nous rapportons un cas de paludisme congénital grave chez un nouveau-né hospitalisé à l'unité de néonatologie de l'Hôpital du Mali.

Observation : Il s'agissait d'un nouveau-né prématuré de 21 jours d'âge corrigé, de sexe féminin qui était hospitalisé pour hyperthermie. Sa mère âgée de 38 ans était une primigeste, primipare. La grossesse était mal suivie. Elle avait pris 2 doses de sulfadoxine/pyriméthamine pour la 
chimioprophylaxie saisonnière contre le paludisme. L'accouchement a été faite par césarienne à 31 $\mathrm{SA}+2$ jours pour procidence du cordon de $3^{\text {è }}$ degré. Le nouveau-né n'a pas été réanimé, il pesait $1600 \mathrm{~g}$ avec une taille à $39 \mathrm{~cm}$ et un périmètre crânien à 30 $\mathrm{cm}$. La maman a présenté une fièvre au post partum immédiat qui a été traité avec artémether/luméfantrine avec succès. Le début de la maladie remontait à une semaine avant l'hospitalisation marqué par une fièvre et anorexie. Elle a été traitée pour infection néonatale la ceftriaxone en raison de $100 \mathrm{mg} / \mathrm{Kg} / \mathrm{j}$ en IVD pendant 7 jours sans succès. Devant la persistance de la fièvre et l'adjonction du coma, de l'hémoglobinurie et de la déshydratation qu'elle a été référée pour une prise en charge. A l'admission, elle pesait $2600 \mathrm{~g}$. Elle avait une hyperthermie à $40^{\circ} \mathrm{C}$ et un mauvais état général. Elle avait une pâleur marquée et un ictère intense. Il y avait des plis de déshydratation qui s'effaçaient lentement. Elle présentait une polypnée superficielle à 65 cycles/mn avec une saturation en oxygène à $90 \%$ à l'air ambiant. Le murmure vésiculaire était bien perçu dans les deux champs pulmonaires. Les bruits du cœur étaient réguliers avec une tachycardie à 180 battements/mn et un souffle systolique intense au foyer mitral. L'abdomen était souple avec une splénomégalie à $5 \mathrm{~cm}$ du rebord costal. La conscience était altérée avec un sore de Blantyre à $3 / 5$. Les réflexes archaïques étaient abolis. La numération formule sanguine a montré une anémie à $2.6 \mathrm{~g} / \mathrm{dl}$ normochrome normocytaire avec une hyperleucocytose à $3700010^{3} / \mu 1$ à prédominance neutrophile. La CRP était négative au latex. La bilirubine totale était à $810,8 \mu \mathrm{mol} / \mathrm{L}$ et la bilirubine indirecte à $721,2 \mu \mathrm{mol} / \mathrm{L}$. Devant cette anémie hémolytique fébrile avec hémoglobinurie un test de
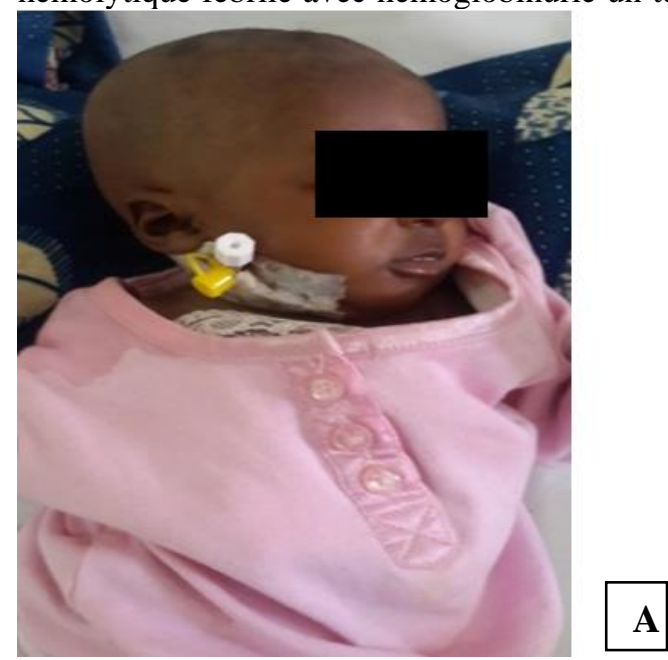

diagnostic rapide du paludisme a été pratiqué en urgence. Il est revenu positif (figure 1).

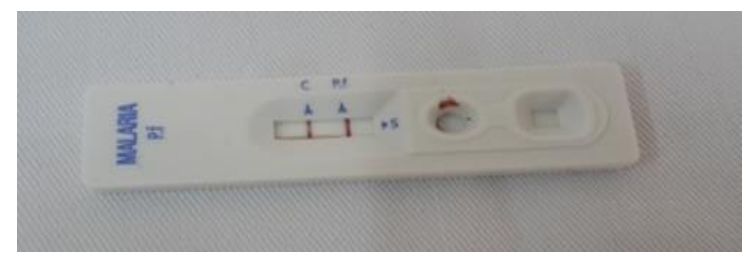

Figure 1 : résultat du test de diagnostic rapide du paludisme

La goutte épaisse a confirmé le paludisme avec une parasitémie à 123000 trophozoïtes par champ de Plasmodium falciparum. Le traitement consistait à une oxygénothérapie 2 à $3 \mathrm{~L}$ par $\mathrm{mn}$, une réhydratation parentérale alternant sérum salé isotonique et Ringer lactate, du paracétamol à raison de $15 \mathrm{mg} / \mathrm{kg}$ toutes les 6 heures, une transfusion de concentré globulaire $\mathrm{O}$ positif et le traitement spécifique antipaludique par artésunate pendant 7 jours selon le protocole du service. $\mathrm{Au}$ bout de 24 heures de traitement l'évolution a été marquée par une apyrexie. La photothérapie intensive a été entamée après l'apyrexie a amélioré l'ictère. $\mathrm{Au} 5^{\mathrm{è}}$ jour d'hospitalisation le nouveau-né est sorti du coma. Au bout de 72 heures de traitement, sur le plan biologique, l'évolution a été marquée par la correction de l'anémie et la négativation de la goutte épaisse. Les bilans rénal et hépatique étaient normaux. Elle est sortie au $7^{\mathrm{è} \mathrm{jour}}$ d'hospitalisation. A la sortie, elle était apyrétique, consciente, reflexes archaïques normaux et pesait $2750 \mathrm{~g}$ avec une splénomégalie à $3 \mathrm{~cm}$ (Figue $2 \mathrm{~A}$ et B).

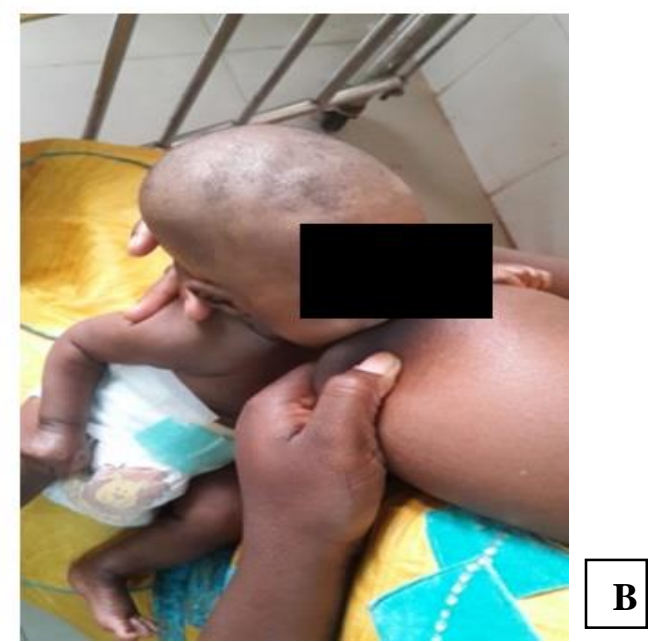

Figure 2 A et $\mathbf{B}$ : photo à la sortie après une semaine d'hospitalisation

Première visite 1 mois après sa sortie poids : $4920 \mathrm{gs}$ PC : $38 \mathrm{~cm}$ Taille : $56 \mathrm{~cm} \mathrm{~T}^{\circ}: 37.01^{\circ} \mathrm{C}$ examen clinque normal en dehors d'une splénomégalie à $3 \mathrm{~cm}$ (figure 3 ). 


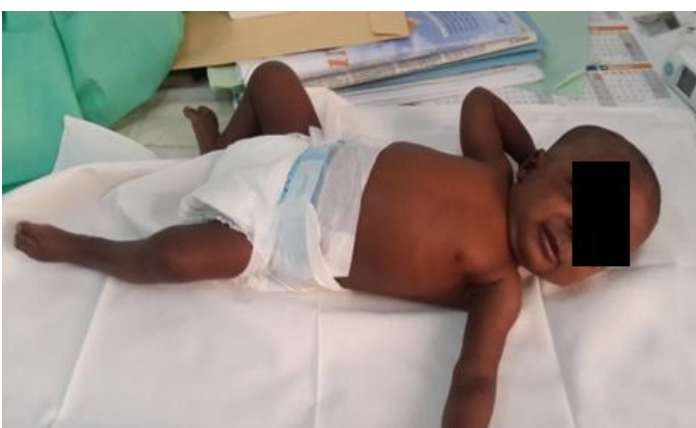

Figure 3 : photo 1 mois après la sortie Discussion

Le paludisme congénital (PC) est une infection transmise de la mère au fotus par voie transplacentaire et concerne les 4 espèces de plasmodies, mais essentiellement le Plasmodium falciparum. Le placenta est une véritable zone d'accumulation du parasite. L'infection placentaire est présente chez le quart de femmes impaludées. La présence ou l'absence des symptomatologies chez un nouveau-né atteint de PC définit respectivement un PC maladie te un PC infection[1].Le paludisme congénital est rare. Pourtant la femme enceinte présente une susceptibilité particulière au paludisme. La prévalence des placentites palustres est supérieure à $30 \%$ en zone holo hypoendémie. Le placenta est en effet, pour des raisons encore mal connues (rôle de l'expression de la chondroïtine sulfate), un site favorable pour la schizogonie. Néanmoins l'infection est rarement symptomatique chez le nouveau-né, le parasite disparait le plus souvent quelques jours $\mathrm{du}$ sang périphérique sans traitement[7]. Cette résistance naturelle du nouveau-né à l'infection palustre repose sur deux facteurs essentiels : La transmission transplacentaire des anticorps maternels; la présence de l'hémoglobine $\mathrm{F}$ défavorable à la croissance du Plasmodium falciparum dans les hématies expliquerait la rapide disparition des parasites chez le nouveau-né et les faibles densités parasitaires lors des infections palustres des premiers mois[7]. Le paludisme congénital (PC) semble exceptionnel en zone d'endémie. Il s'agit presque constamment de PC infection; les cas de PC maladie sont essentiellement décrits en zone non endémique. Ils sont diagnostiqués dans $24 \%$ des cas à la naissance et dans $52 \%$ des cas après le 14 è jour de vie[7]. La symptomatologie n'est pas spécifique. Les signes cliniques motivant la goutte épaisse sont la fièvre : 75\% ; splénomégalie 33\%; hépatomégalie : $16 \%$; anémie :11\% ; pâleur, ictère, anorexie $: 7,7 \%[1]$. La parasitémie éventuellement observée à la naissance a une faible densité qui peut être responsable d'une goutte épaisse négative. Elle a aussi une grande fugacité et une faible vitalité car la goutte épaisse est le plus souvent négative au $2^{\mathrm{e}}$ $3^{3}$ jour[1]. Le traitement des formes graves et compliquées repose sur les antipaludéens par voie parentérale pendant 5-7 jours. Il n'existe pas de données parfaitement établies par rapport à l'utilisation de l'artésunate dans le traitement du paludisme congénital grave. Le groupe de travail de l'OMS considère que la quinine ou l'artesunate peuvent indifféremment être utilisés dans le traitement du paludisme grave avant 18 mois[8].

\section{Conclusion}

On retiendra l'intérêt d'un test de diagnostic rapide du paludisme dans une anémie hémolytique du nouveau-né dans les zones d'endémie palustre, l'insuffisance des 2 doses sulfadoxine pyriméthamine dans le traitement préventif intermittent et l'efficacité et la bonne tolérance de l'artésunate dans le traitement du paludisme grave du nouveau-né.

\section{Déclaration d'intérêts}

Nous, auteurs déclarons ne pas avoir de conflits d'intérêts en rélation avec cet article.

\section{Références}

1. 1. Aujard Y. Infections néonatales bactériennes, mycosiques et parasitaires. In : Encyl Med Chir Pédiatrie. Elsevier Masson. Paris ; 2011. p. 15. (Elsevier Masson).

2. 2.OMS $\mid 10$ menaces sur la santé mondiale en 2018 [Internet]. [Cité 17 avr 2018].

3. 3.Programme National de Lutte contre le paludisme. Enquête sur les Indicateurs du Paludisme au Mali. Bamako : Ministère de la Santé de l'Hygiène Publique ;2016 juill p. 2.

4. 4. Diouf FN. Prévalence du paludisme congénital infestation a plasmodium falciparum au centre Hospitalier Régional de Ziguinchor/Sénégal. CAMES SANTE. 2015;3(1).

5. 5.Kaboré W E. Paludisme congénital en 2013 dans le département de pédiatrie du Centre Hospitalier Universitaire Souro Sanou: à propos de 2 cas. [Thèse Med]: Université Polytschnique de Bobodioulasso; 2013.

6. 6.F Dicko-Traoré, Sylla M, Diakité A Z et al. Le paludisme congénital et néonatal en Afrique subsaharienne, un évènement rare? Journal de Pédiatrie et Puériculture 2011;24(2):57-61.

7. 7.Imbert P, Banerjee A. Paludisme de l'enfant. In : EMC pédiatrie/Maladies infectieuses. Scientifiques et Médicales Elsevier. Paris ; 2002. p. 24. (Elsevier Masson).

8. Comission Spécialisée Maladies Transmissibles. Place de l'artesunate injectable dans le traitement du paludisme grave chez l'adulte et l'enfant. Paris : Haut Conseil de la Santé Publique; 2013 févr p. 17-20. (Rapport du groupe de travail). 